\title{
Specifics of Platelet Hemostasis in Children with Chronic Diseases of Lungs
}

\author{
Olga B. Gordeeva, Victoria V. Botvinieva, Olga I. Simonova, Leyla S. Namazova-Baranova, \\ Yulia V. Gorinova, Nato D. Vashakmadze, Anna K. Gevorkyan \\ Federal State Budgetary Scientific Institution "Scientific Centre of Children Health", Moscow, Russia \\ Email: obr@yandex.ru
}

Received 9 April 2016; accepted 24 April 2016; published 28 April 2016

Copyright (C) 2016 by authors and OALib.

This work is licensed under the Creative Commons Attribution International License (CC BY). http://creativecommons.org/licenses/by/4.0/

(c) (i) Open Access

\section{Abstract}

Study of platelet aggregation function can help early diagnostics of microvasculature's disorders. Purpose of the study is evaluation of changes of platenet aggregation activity in children with chronic inflammation. Materials and methods: 85 children examined, of whom 45 children with cystic fibrosis (mixed form) aged 8 months to 17 years. The platelet aggregation in children's whole blood was studied using aggregometer "Multiplate" (VerumDiagnostica, Germany). The following agents were used as inducer of aggregation: adenosine diphosphoric acid solution (ADP), soluble Thrombin Receptor Activator for Peptide 6 (TRAP-test) and arachidonic acid (ASPI-test). $C$-reactive protein (SRP) in the blood serum was measured by turbidimetric method using biochemical analyzer UniCelDxC 600 (BD, USA). Immunochemiluminescent method was used to determine the level of the main pro-inflammatory cytokine IL-6 (analyzer Accesses 2, BD, USA). Results: changes in the aggregation of platelets can be expressed by platelet activation followed by hyper-aggregation and thrombi formation on the one hand, and disorder of arachidonic acid and ADP metabolism on the other hand that leads to the development of hemorrhagic complications. A necessary condition of the therapy is a presence of antiplatelets and calcic drugs, which help to warn complications in a form of thrombosis and hemorrhages.

\section{Keywords}

Platelet Aggregation Function, Cystic Fibrosis, Inflammation, Children

Subject Areas: Pediatrics

\section{Introduction}

Cystic fibrosis (CF) is a monogenic disease caused by mutation in the CFTR gene (cystic fibrosis transmembrane conductance regulator); the disease is characterized by damage of all exocrine glands of vital organs [1].

How to cite this paper: Gordeeva, O.B., Botvinieva, V.V., Simonova, O.I., Namazova-Baranova, L.S., Gorinova, Y.V., Vashakmadze, N.D. and Gevorkyan, A.K. (2016) Specifics of Platelet Hemostasis in Children with Chronic Diseases of Lungs. Open Access Library Journal, 3: e2324. http://dx.doi.org/10.4236/oalib.1102324 
According to recent data, the inflammation process always involves microvasculature [2] [3]. A number of pathological changes in the process of inflammation affect the endothelium of pulmonary vessels, which, in turn, inactivates serotonin, prostaglandins and bradykinin and synthesizes factors coagulation and anticoagulation systems. Subsequently, the dysfunction of endothelium may cause the development of vascular complications.

Because of the fact that chronic inflammation develops in conditions of mucociliardisfunction of lungs, it leads to changes in capacity of blood cells. It is known that platelets play an important role in inflammatory responses' cascade. Changes of platelet aggregation function may cause to development of both thrombosis and hemorrhages. Therefore, in our opinion, the study of platelet stage of hemostasis can facilitate early detection of disorders in microvasculature and their timely correction.

The purpose is to evaluate changes of platelet stage of hemostasis in children with cystic fibrosis.

\section{Materials and Methods}

The study was approved by a local Ethics Committee. Follow-up prospective study of 85 children was undertaken, 45 children of them were with cystic fibrosis (mixed form) aged 8 months to 17 years. The median age was $7.57 \pm 4.66$ years. The mean Shvakhman-Brasfild score was $52.3 \pm 1.1$. The diagnosis "cystic fibrosis" was made on the basis of positive sweat test and confirmed by the results of genetic tests. All patients were followed in the acute phase of the bronchopulmonary process (increased cough, profuse mixed moist rales, large amount of purulent viscous mucus, negative dynamics of X-ray pattern) and were receiving background therapy: antibiotics (III and IV generation cephalosporins, carbapenems) in maximum dosage; drugs of N-acetylcysteine group and ambroxol hydrochloride, mucolytic with anti-inflammatory properties—Dornase-alpha (Pulmozyme, F. Hoffmann-La Roche Ltd, Switzerland). The comparison group consisted of 40 healthy children of the same age, who passed clinical examination at Consultation and Diagnostic Clinic of the "Scientific Center of Children Health". All patients underwent blood tests (EDTA stabilized blood) using blood analyzer Sysmex 200i (Japan). The platelet aggregation in children's whole blood was studied using aggregometer "Multiplate" (VerumDiagnostica, Germany). The following agents were used as inducer of aggregation: adenosine diphosphoric acid solution (ADP), soluble Thrombin Receptor Activator for Peptide 6 (TRAP-test) and arachidonic acid (ASPI-test). C-reactive protein (SRP) in the blood serum was measured by turbidimetric method using biochemical analyzer UniCelDxC 600 (BD, USA). Immunochemiluminescent method was used to determine the level of the main pro-inflammatory cytokine IL-6 (analyzer Accesses 2, BD, USA). The data were processed using nonparametric methods of variation statistics, Mann-Whitney test for comparison of groups (SPSS 20.0 software). The results were considered as significant with significance level $\mathrm{p}<0.05$. Also ROC analysis of examined indicators was made (program SPSS 20.0, IBM corp.USA).

\section{Results}

Based on the obtained indicators of the primary stage of hemostasis, statistically significant abnormalities of aggregation of platelets were found in 2/3 of patients with mixed form of cystic fibrosis (as compared with the control group, $\mathrm{p}<0.05)$. See Table 1.

In the study of aggregation with ADP and arachidonic acid, the trend of hypo-aggregation of platelets was noted in 40 percent of patients. When analyzing the condition of thrombin receptors on the platelet surface, the increased rate and degree of thrombin-induced aggregation was found in 22.2 percent of children with cystic fi-

\begin{tabular}{cccc} 
Table 1. Blood value medians. & & & \\
\hline $\begin{array}{c}\text { Lab blood values } \\
\text { 95 DI }\end{array}$ & Cystic fibrosis group & Control group & P Significance value \\
\hline TRAP test (U) & $83(8.5-132.85)$ & $55(35-82.7)$ & 0.001 \\
ASPI test (U) & $42.5(10-102.35)$ & $51.5(31-80.75)$ & 0.235 \\
ADF test (U) & $38.5(7.25-80)$ & $45(26-71.3)$ & 0.042 \\
IL-6 pg/ml & $8.3(2.07-45.18)$ & $0.2(1.38-4.59)$ & 0.001 \\
CRP mg/l & $0.1(0.1-27.36)$ & $0.1-1.28)$ & 0.011 \\
\hline
\end{tabular}


brosis. Combined abnormalities in aggregation of platelets were found in one third of patients. The most pronounced changes in platelet function were in the form of thrombin-induced hyper-aggregation and ADP-hypoaggregation in CF patients during acute phase of broncho-pulmonary process; it was accompanied by increased levels of serum IL-6 (Figure 1). Hyper-aggregation with thrombin receptor activator (Figure 2) in children with CF may indicate abnormalities in the primary hemostasis with the tendency to thrombosis. Hypo-aggregation with ADP (Figure 3) in children with CF reflects a delayed ADP release reaction in platelets that signals a decrease in platelet aggregation and the risk of bleeding. Along with the indicators of platelet aggregation activity, we studied the WBC and platelets counts, platelet indices, and the levels of inflammatory markers in blood serum: CRP, IL-6. In CF patients, statistically significant differences were detected in two platelet aggregation tests: TRAP and ADP, as well as in terms of levels of serum inflammation markers IL-6 and CRP $(\mathrm{p}<0.05)$. The values of platelet indices, platelet count and platelet aggregation test with arachidonic acid did not differ significantly from those of healthy children $(p>0.05)$.

In order to identify the most informative diagnostic tests of the primary stage of hemostasis and inflammatory

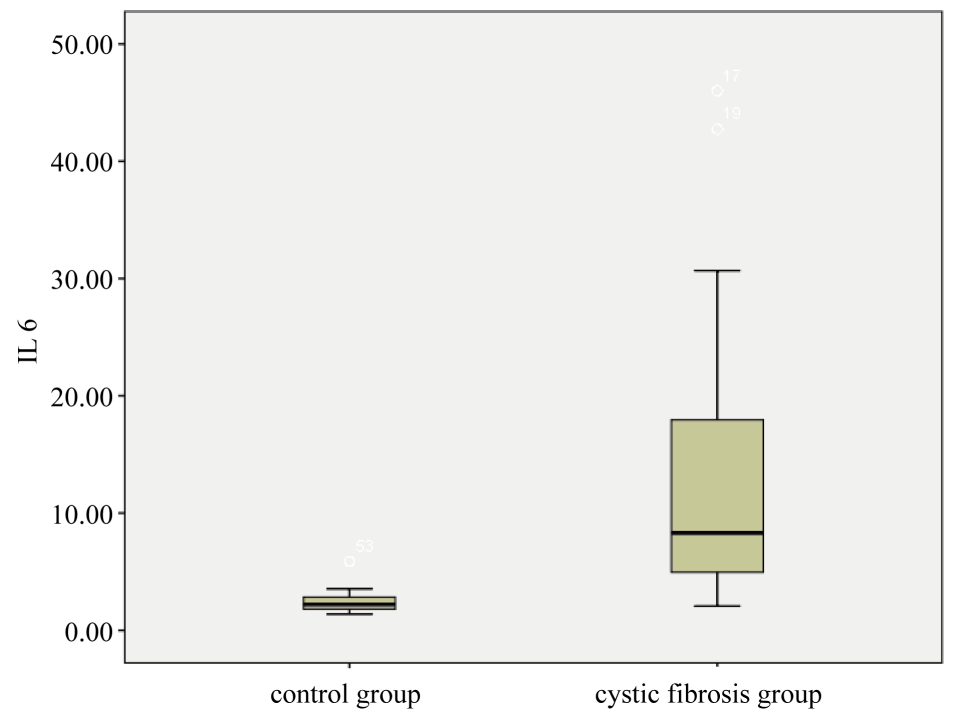

Figure 1. The values of serum IL-6 (ng/mL).

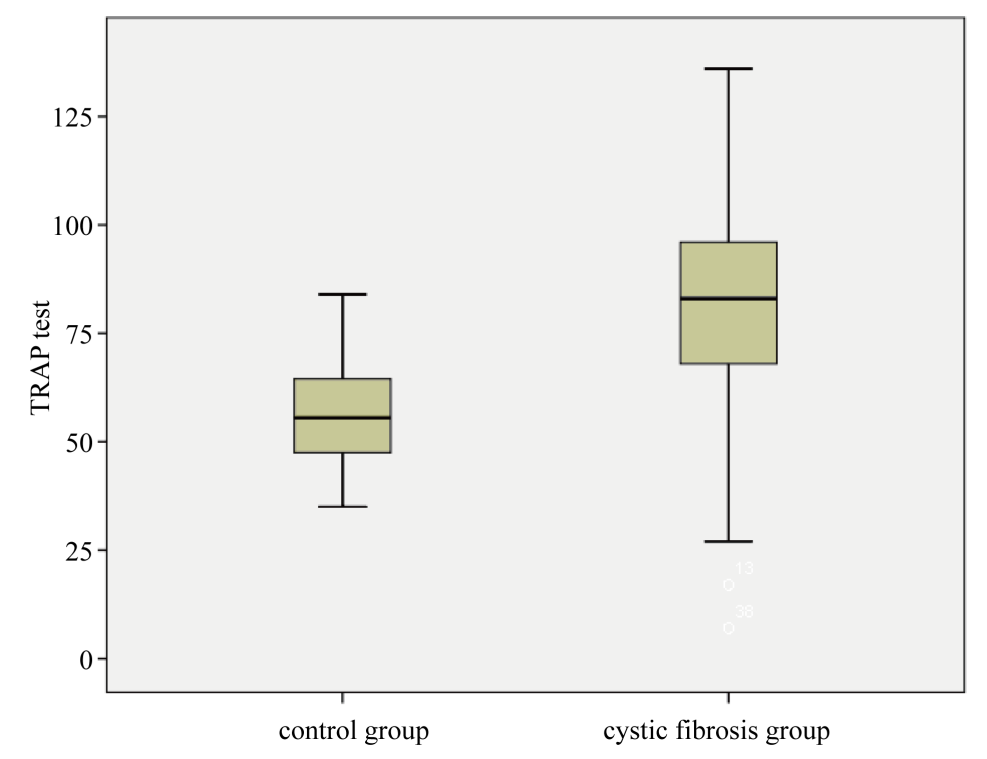

Figure 2. Platelet aggregation with TRAP. 


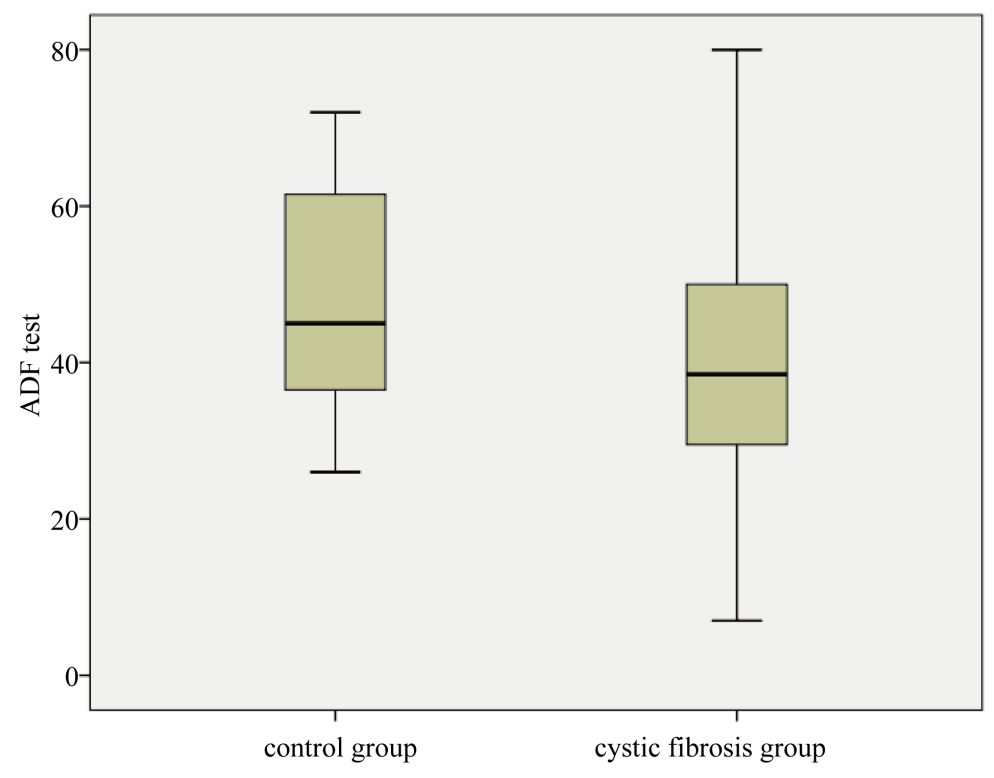

Figure 3. Platelet aggregation with ADP.

markers in case of cystic fibrosis, we further analyzed ROC-curves using the obtained data. TRAP-test has the best diagnostic value among the tests that reflect the aggregation function of platelets (Figure 4), and IL-6 is the best among the serum markers of inflammation (Figure 5). Quantitative interpretation of ROC analysis in terms of AUC (areaunder ROC curve) is shown in Table 2.

\section{Discussion}

Previously studies of platelet aggregation function were pursued under thrombocytopenic purpura and thrombocytopenia, also under impaired fat metabolism of children and teenagers with overweight [3]. It has been established that children with overweight have platelet hyper-aggregation. Also there are earlier studies concerning appraised value of inhibitory influence of prostaglandin PGE1 on ADP.

In some works researches on platelet activity were carried out by flow cytometry [4]. It was found that by exacerbation of inflammation in the setting of cystic fibrosis amount of circulative active platelets grows up alongside with monocyte-derived thrombocytic and neutrophilousthrombocytic cell pool.

Also there are some works [5] concerning value of MPV criteria change by exacerbation of inflammation in the setting of cystic fibrosis, which can be predictor of exacerbation.

We have not met works similar to our study in accessible bibliography. We pursued researches of platelet aggregation function in the setting of change of inflammatory markers present at chronic diseases of lungs.

Obtained data suggest that in children suffering cystic fibrosis, against the background of acute bronchopulmonary process, platelets are involved in the inflammatory response. Changes in the aggregation of platelets can be expressed by platelet activation followed by hyper-aggregation and thrombi formation on one hand, and disorder of arachidonic acid and ADP metabolism on the other that leads to the development of hemorrhagic complications. We believe that abnormalities in the primary stage of hemostasis in children with CF deserve further investigation with other inducers of aggregation with the purpose to prevent the development of hemorrhagic and thrombotic complications and adequate therapeutic correction of disorders in the primary stage of hemostasis.

\section{Conclusion}

Different specifics of platelet aggregation-hyper- and hypo-aggregation-have been found out, which testify to increased or decreased platelet activity with given group of patients. Taking into account specifics of clinical aspects and development of possible complications in platelet hemostasis, we consider that a necessary condition of the therapy is the presence of antiplatelets and calcic drugs, which helps to warn complications in a form 


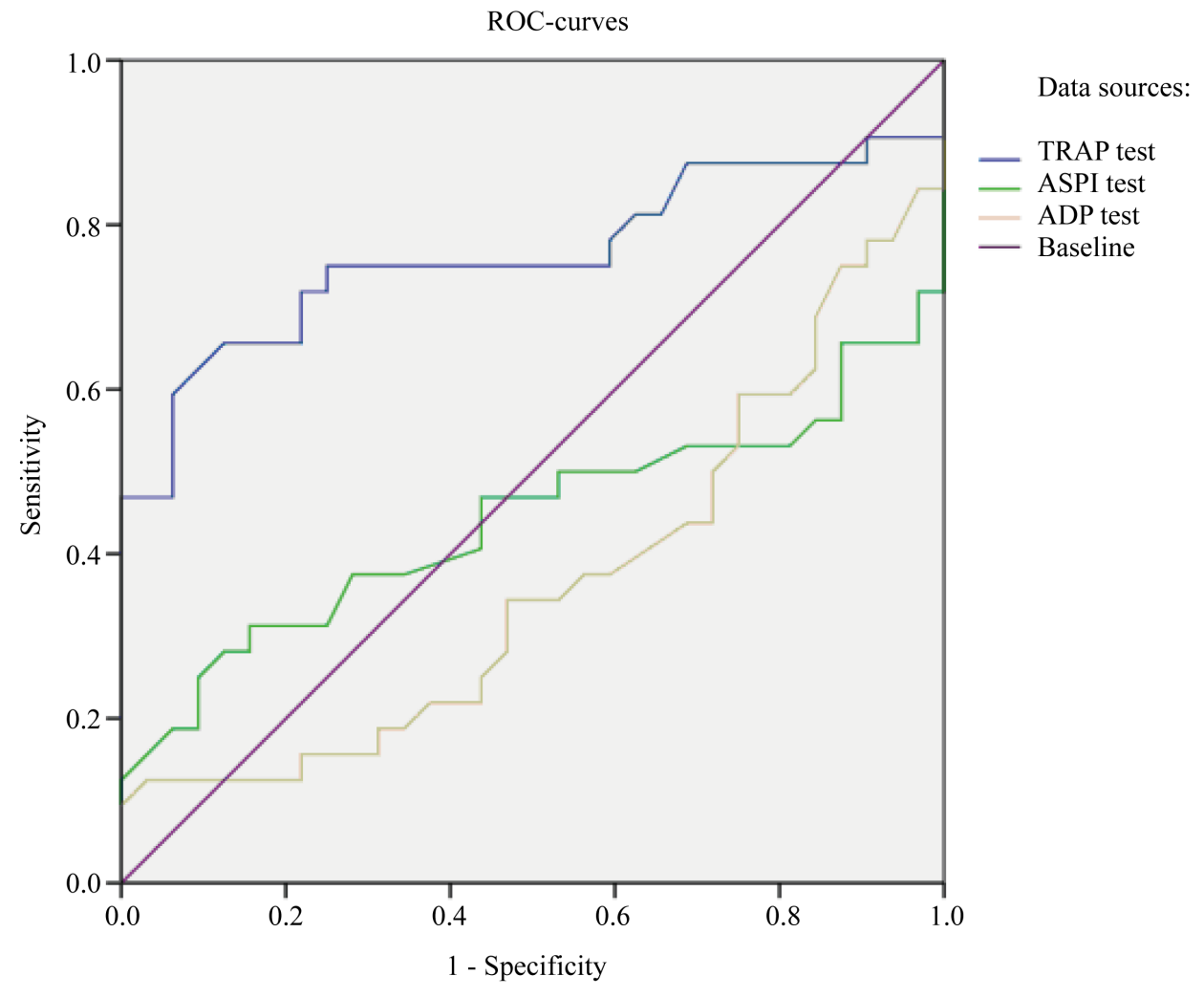

Figure 4. ROC-curves for platelet aggregation tests.

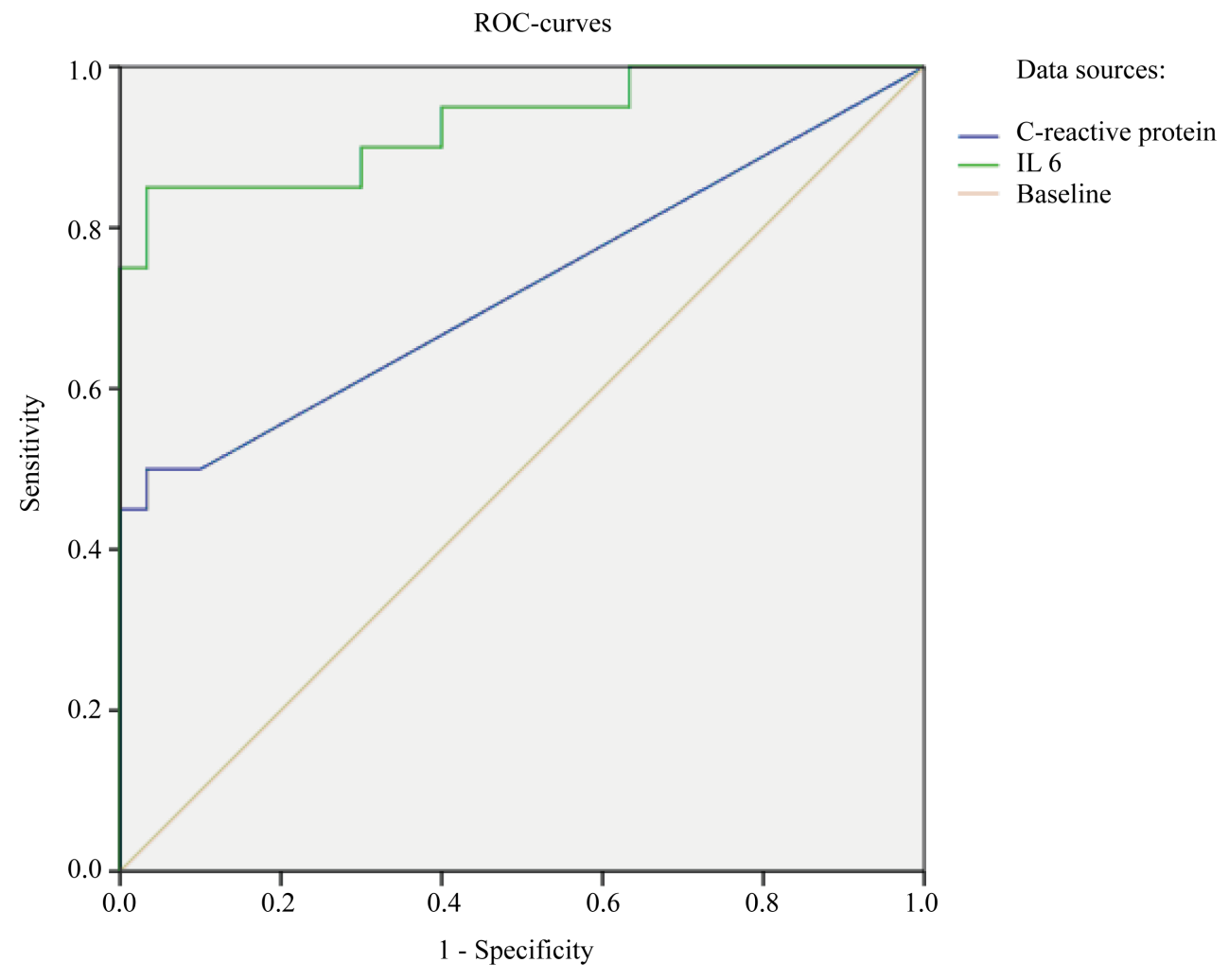

Figure 5. ROC-curves for serum inflammation markers. 
Table 2. Clinical significance of tests (ROC analysis).

\begin{tabular}{ccccc}
\hline \multirow{2}{*}{$\begin{array}{c}\text { Test parameter } \\
\text { Area under the curve (AUC) }\end{array}$} & SD & \multicolumn{2}{c}{ Asymptotic 95\% confidence interval } \\
\cline { 4 - 5 } C-reactive protein & 0.726 & 0.109 & Lower limit & Upper limit \\
IL6 & 0.908 & 0.061 & 0.512 & 0.940 \\
TRAP test & 0.812 & 0.107 & 0.789 & 1.000 \\
ASPI test & 0.522 & 0.137 & 0.602 & 0.791 \\
ADP test & 0.330 & 0.094 & 0.253 & 0.514 \\
\hline
\end{tabular}

of thrombosis and hemorrhages.

\section{References}

[1] Hodson, M. and Geddes, D. (1995) Cystic Fibrosis. Chapman, London, 439.

[2] Vernooy, J.H., Kucukaycan, M. and Jacobs, J.A. (2002) Local and Systemic Inflammation in Patients with Chronic Obstructive Pulmonary Disease: Soluble Tumor Necrosis Factor Receptors Are Increased in Sputum. American Journal of Respiratory and Critical Care Medicine, 166, 1218-1224. http://dx.doi.org/10.1164/rccm.2202023

[3] Lohse, J., Schweigel, J., Naeke, A., Lee-Kirsch, M.A., Siegert, G., Bergmann, S., Kuhlisch, E., Suttorp, M. and Knöfler, R. (2010) Platelet Function in Obese Children and Adolescents. Hamostaseologie, 30, 126-132.

[4] O’Sullivan, B.P., Linden, M.D., Frelinger, A.L., Barnard, M.R. and Spencer-Manzon, M. (2005) Platelet Activation in Cystic Fibrosis. Blood, 105, 4635-4641. http://dx.doi.org/10.1182/blood-2004-06-2098

[5] Uysal, P., Tuncel, T., Olmez, D., Babayigit, A., Karaman, O. and Uzuner, N. (2011) The Role of Mean Platelet Volume Predicting Acute Exacerbations of Cystic Fibrosis in Children. Annals of Thoracic Medicine, 6, 227-230. http://dx.doi.org/10.4103/1817-1737.84778 